\title{
Transmit Beamforming for Space-Frequency Coded MIMO-OFDM Systems with Spatial Correlation Feedback
}

\author{
Ahmed K. Sadek, Member, IEEE, Weifeng Su, Member, IEEE, and K. J. Ray Liu, Fellow, IEEE
}

\begin{abstract}
This paper addresses the problem of joint optimization of transmit beamforming and space-frequency (SF) coding for MIMO-OFDM systems with spatial correlation feedback in broadband communications. This problem is challenging in the sense that the transmitter should be designed to beamform across multiple eigenspaces associated with the multipath environment simultaneously. With arbitrary transmit spatial correlation, the performance analysis for SF-coded MIMO-OFDM systems with beamforming is provided, and a general optimization problem for the beamforming design is formulated. Three suboptimal approaches to design the beamformer based on the derived design criteria are proposed: i) Eigenvalue selection scheme; ii) Eigenspace selection scheme; and iii) Per-subcarrier approach based on decoding at each subcarrier. The proposed schemes take into account the multiple eigenspace information associated with the multipath-delay channel. Improvement in the performance over SF coding without beamforming is shown through simulations in terms of bit error rate. The Eigenvalue selection scheme provides the best performance among the proposed algorithms. This scheme locates the subspace associated with the largest eigenvalues in the eigenspace of the covariance matrices. With the Eigenvalue selection scheme, the performance improvement is about $3 \mathrm{~dB}$ over the SF coding without beamforming for highly correlated channels as shown in our simulations.
\end{abstract}

Index Terms-Broadband wireless communications, MIMOOFDM systems, transmit beamforming across multiple eigenspaces, multiple antennas, space-frequency coding, correlation feedback.

\section{INTRODUCTION}

D ESIGNING transmit-diversity schemes for multipleinput-multiple-output (MIMO) systems depends on the form of channel state information (CSI) available at the transmitter. With no CSI, space-time coding can achieve the diversity gain inherent in MIMO flat-fading channels [1], [2]. On the other hand, with perfect CSI transmit beamforming maximizes the signal-to-noise-ratio at the receiver side [3], [4]. For the more general case, the transmitter can acquire

Paper approved by R. W. Heath, Jr., the Editor for MIMO Techniques of the IEEE Communications Society. Manuscript received August 16, 2005; revised June 8, 2006 and February 9, 2007. This work was supported in part by U.S. Army Research Laboratory under Cooperative Agreement DAAD 190120011.

A. K. Sadek is with Corporate R\&D, Qualcomm Incorporated, San Diego, CA 92121, USA (e-mail: asadek@qualcomm.com).

W. Su is with the Department of Electrical Engineering, State University of New York at Buffalo, USA (e-mail: weifeng@eng.buffalo.edu).

K. J. Ray Liu is with the Department of Electrical and Computer Engineering, and the Institute for Systems Research, University of Maryland, College Park, MD 20742, USA (e-mail: kjrliu@eng.umd.edu).

Digital Object Identifier 10.1109/TCOMM.2008.050237 partial information via a feedback channel from the receiver or during a duplex mode of transmission. In literature, two main categories of channel feedback are considered [5], [6]: i) mean feedback: where the transmitter attains an estimate of the current channel realization; and ii) covariance feedback: the transmitter knows the spatial covariance structure of the channel.

Extensive studies have been done in literature for MIMO communication systems over flat fading channels with various channel feedback information [5]-[8]. For the case of frequency selective fading channels few works have investigated the effect of the CSI feedback on MIMO-OFDM systems [4], [9], [10], [13], [14]. In [4], the authors assumed perfect CSI at the transmitter, and they proposed a joint transmitreceive beamforming design for multicarrier frequency selective fading MIMO systems. A mean feedback model was adopted in [9], and an adaptive two-dimensional space-time coded beamformer over each subacarrier was developed. Note that utilizing space-time coding on each subcarrier cannot, in general, exploit the frequency diversity available in the frequency selective fading environment [11]. To reduce feedback overhead in mean-feedback scenarios, [12] proposes a beamforming technique where the receiver utilizes quantized feedback. In [13], eigenbeamforming with selection diversity is investigated where eigenmodes are selected to maximize the instantaneous received SNR for each subcarrier. In [14], and under an information theoretic framework, an efficient technique to get covariance channel information at the transmitter is proposed where the covariance matrix for the downlink is obtained by suitably averaging uplink channel measurements across frequency.

In the previous work discussed above, perfect CSI and mean feedback might not be feasible in mobile scenarios where the channel is quickly varying. As a result, covariance feedback becomes an adequate approach to adopt as the channel statistics do not vary quickly. In this paper, we consider the problem of transmit beamforming design for MIMO-OFDM systems when the covariance matrix of the channel is available at the transmitter. Our major contribution compared to previous work is that we formulate an optimization problem to design a beamformer jointly for all subcarriers in a MIMO-OFDM system, and we show the criteria for optimal design. We further develop suboptimal approaches to design the beamformer and show by simulations the improvement in performance compared to SF coding without beamforming. 
In particular, we derive the average pairwise error probability of a MIMO-OFDM system with arbitrary transmit spatial correlation, and we formulate a general beamformer optimization problem in terms of minimizing the average pairwise error probability. For this joint optimization problem, we provide the criteria to design a SF-beamforming scheme. Then, we adopt a transmitting scheme in which we utilize a SF code that can achieve full diversity for uncorrelated channel scenarios and design a beamformer that enhances the performance of the code under the knowledge of the channel covariance structure. In a flat fading scenario, the problem has a closed form solution, which results in a space-time beamformer design [8]. However, in a MIMO multipath environment, there exist in general $L$ delay paths. As a result, the eigenspace associated with the channel covariance matrices is $L$ times larger than that of the beamformer, i.e., the beamformer has to match to multiple eigenspaces simultaneously. This is different from the MIMO flat fading case, where the number of degrees of freedom available to design the beamformer is equal to the dimension of the channel covariance matrix that the beamformer should match to.

The performance analysis of the system reveals that it is difficult, if not impossible, to find a closed form solution for designing the optimum beamformer for MIMO-OFDM systems. Based on the average pairwise error probability, we propose three suboptimal approaches to design the beamformer. Simulations are conducted to compare the performance of the proposed algorithms to SF coding without beamforming and instantaneous beamforming.

\section{SYSTEM MODEL}

We consider a MIMO frequency selective fading channel model with $M_{t}$ transmit antennas and $M_{r}$ receive antennas. OFDM with $N$ subcarriers is utilized, as it provides an attractive means to lower the complexity of equalization and decoding in frequency selective environment [15]. The multipath channel has $L$ significant delay paths between each transmit-receive antenna pair. The path gains for different delays are assumed to be independent. The channel impulse response from transmit antenna $i$ to receive antenna $j$ can be modeled as

$$
h_{i j}(\tau)=\sum_{l=0}^{L-1} \alpha_{i j}(l) \delta\left(\tau-\tau_{l}\right),
$$

where $\tau_{l}$ is the delay of the $l$-th path, and $\alpha_{i j}(l)$ is the complex path gain between transmit antenna $i$ and receive antenna $j$. The $\alpha_{i j}(l)$ are modeled as zero mean, circularly symmetric complex Gaussian random variables with variance $\beta_{l}^{2}$. The channel gains are assumed jointly Gaussian. The time delay $\tau_{l}$ and the variance $\beta_{l}^{2}$ are the same for each transmit receive link [16]. The power of the $L$ paths are normalized such that $\sum_{l=0}^{L-1} \beta_{l}^{2}=1$. From (1), the frequency response of the channel is given by $\tilde{H}_{i j}(f)=\sum_{l=0}^{L-1} \alpha_{i j}(l) e^{-\mathbf{j} 2 \pi f \tau_{l}}$, where $\mathbf{j}=\sqrt{-1}$. We consider spatial correlation at the transmitter side, while different receive antennas are assumed independent and have the same fading statistics, i.e.,

$$
E\left[\alpha_{i j}(l) \alpha_{p q}^{*}(l)\right]=\beta_{l}^{2} r(i-p) \delta(j-q),
$$

where $r(i-p)$ is the spatial correlation factor between transmit antennas $i$ and $p$, and $\delta(\cdot)$ is the delta function. This model generally arises when the transmitter is unobstructed with many scatterers, while the receiver is surrounded by a rich scattering environment, as in the downlink of a cellular system.

At the transmitter, the input bits to the SF-beamformer coder are divided into $b$ bits-long, which are then mapped into a SF-beamformer symbol. Each SF-beamformer symbol can be expressed as an $M_{t} \times N$ matrix

$$
\mathbf{B}=\left[\begin{array}{llll}
\mathbf{b}(0) & \mathbf{b}(1) & \ldots & \mathbf{b}(N-1)
\end{array}\right],
$$

where $\mathbf{b}(n)=\left[\begin{array}{llll}b_{1}(n) & b_{2}(n) & \ldots & b_{M_{t}}(n)\end{array}\right]^{T}$ is an $M_{t} \times$ 1 column vector. The SF-beamformer symbol $\mathbf{B}$ is assumed to satisfy the energy constraint $E\left[\|\mathbf{B}\|_{F}^{2}\right]=N M_{t}$, where $E[\cdot]$ denotes expectation, and $\|\mathbf{B}\|_{F}$ is the Frobenius norm of $\mathbf{B}$. The OFDM transmitter applies IFFT to each row of the matrix B. By appending a cyclic prefix, it transmits the OFDM symbol corresponding to the $i$-th row of $\mathbf{B}$ at the $i$-th antenna.

At the receiver, after matched filtering, removing the cyclic prefix, and applying FFT, the received signal at the $n$-th subcarrier at receive antenna $j$ is given by

$$
y_{j}(n)=\sqrt{\frac{\rho}{M_{t}}} \mathbf{h}_{j}^{T}(n) \mathbf{b}(n)+v_{j}(n),
$$

where

$$
\mathbf{h}_{j}(n)=\left[\begin{array}{llll}
H_{1 j}(n) & H_{2 j}(n) & \ldots & H_{M_{t} j}(n)
\end{array}\right]^{T},
$$

in which $H_{i j}(n)=\sum_{l=0}^{L-1} \alpha_{i j}(l) e^{-\mathbf{j} 2 \pi n \Delta f \tau_{l}}$, represents the channel frequency response at the $n$-th subcarrier between transmit antenna $i$ and receive antenna $j$, where $\Delta f=1 / T$ is the subcarrier frequency separation, and $T$ is the OFDM symbol period. In (4), $\mathbf{b}(n)$ is the $n$-th column of the matrix $\mathbf{B}$, and it represents the channel symbol vector transmitted on the $n$-th subcarrier. The term $v_{j}(n)$ in (4) denotes the additive white circularly symmetric complex Gaussian noise, with zero mean and unit variance, at the $n$-th subcarrier at receive antenna $j$.

\section{Performance Analysis and General BEAMFORMER DESIGN}

In this section, we analyze the performance of the MIMOOFDM system with arbitrary channel correlation conditions at the transmitter as specified in the previous section. Then, we formulate a general optimization problem to design a joint SF-beamformer symbol.

\section{A. System Performance Analysis}

First we derive an expression for the average pairwise error probability. We rewrite the received signal in (4) in matrix form as

$$
\mathbf{y}=\sqrt{\frac{\rho}{M_{t}}} \mathbf{H} \operatorname{vec}(\mathbf{B})+\mathbf{v},
$$

where the $N M_{r} \times N M_{t}$ channel matrix $\mathbf{H}$ is formatted as $\mathbf{H}=\left[\begin{array}{llll}\mathbf{H}_{1}^{T} & \mathbf{H}_{2}^{T} & \ldots & \mathbf{H}_{M_{r}}^{T}\end{array}\right]^{T}$, in which $\mathbf{H}_{j}$ represents the channel frequency response to receive antenna $j$, and is formatted as an $N \times N M_{t}$ block diagonal matrix as follows 
$\mathbf{H}_{j}=\operatorname{diag}\left(\mathbf{h}_{j}^{T}(0), \mathbf{h}_{j}^{T}(1), \ldots, \mathbf{h}_{j}^{T}(N-1)\right)$. The average pairwise error probability between two channel symbols $\mathbf{B}$ and $\tilde{\mathbf{B}}$ assuming coherent detection can be bounded with [18]

$$
\operatorname{Pr}(\mathbf{B} \rightarrow \tilde{\mathbf{B}}) \leq\left(\frac{\rho}{4 M_{t}}\right)^{-r\left(\mathbf{R}_{\Phi}\right)}\left(\prod_{i=0}^{r\left(\mathbf{R}_{\Phi}\right)-1} \mu_{i}\left(\mathbf{R}_{\Phi}\right)^{-1}\right)
$$

where $\boldsymbol{\Phi}$ is an $N M_{r} \times 1$ vector given by $\boldsymbol{\Phi}=$ $\mathbf{H}[\operatorname{vec}(\mathbf{B})-\operatorname{vec}(\tilde{\mathbf{B}})]$, and $\mathbf{R}_{\boldsymbol{\Phi}}$ is its covariance matrix. $r(\cdot)$ and $\mu_{i}(\cdot)$ denote the rank and $i$-th eigenvalue of a matrix.

The diversity gain of the system is given by $r\left(\mathbf{R}_{\boldsymbol{\Phi}}\right)$, and the coding gain is given by the product term $\prod_{i=0}^{r\left(\mathbf{R}_{\Phi}\right)-1} \mu_{i}\left(\mathbf{R}_{\boldsymbol{\Phi}}\right)$. The covariance matrix $\mathbf{R}_{\Phi}$ can be written as

$$
\begin{aligned}
\mathbf{R}_{\boldsymbol{\Phi}} & =E\left[\mathbf{H v e c}(\mathbf{B}-\tilde{\mathbf{B}}) \operatorname{vec}^{\mathcal{H}}(\mathbf{B}-\tilde{\mathbf{B}}) \mathbf{H}^{\mathcal{H}}\right] \\
& =E\left(\mathbf{H} \boldsymbol{\Delta} \boldsymbol{\Delta}^{\mathcal{H}} \mathbf{H}^{\mathcal{H}}\right),
\end{aligned}
$$

where $\boldsymbol{\Delta}=\operatorname{vec}(\mathbf{B}-\tilde{\mathbf{B}})$. The channel matrix $\mathbf{H}$ contains the channel frequency response from the transmitter to different receive antenna. (8) can be further expanded as

$$
\mathbf{R}_{\boldsymbol{\Phi}}=E\left\{\left[\begin{array}{c}
\mathbf{H}_{1} \boldsymbol{\Delta} \\
\vdots \\
\mathbf{H}_{M_{r}} \boldsymbol{\Delta}
\end{array}\right]\left[\begin{array}{lll}
\boldsymbol{\Delta}^{\mathcal{H}} \mathbf{H}_{1}^{\mathcal{H}} & \ldots & \boldsymbol{\Delta}^{\mathcal{H}} \mathbf{H}_{M_{r}}^{\mathcal{H}}
\end{array}\right]\right\} .
$$

With the assumption that the fading at different receive antennas is independent with identical statistics, we can write (9) as

$$
\mathbf{R}_{\boldsymbol{\Phi}}=\mathbf{I}_{M_{r}} \otimes E\left[\mathbf{H}_{i} \Delta \Delta^{\mathcal{H}} \mathbf{H}_{i}^{\mathcal{H}}\right] .
$$

Note that the term $E\left[\mathbf{H}_{i} \boldsymbol{\Delta} \boldsymbol{\Delta}^{\mathcal{H}} \mathbf{H}_{i}^{\mathcal{H}}\right]$ is independent of the receive antenna, and denote it as $\Psi$. Since, the matrix $\mathbf{H}_{i}$ has a diagonal structure, the matrix $\Psi$ can be expressed as shown in (11), where $\boldsymbol{\Delta}(n)=\mathbf{b}(n)-\tilde{\mathbf{b}}(n)$. Furthermore, the vector $\mathbf{h}_{i}(n)$ can be written as

$$
\mathbf{h}_{i}(n)=\sum_{l=0}^{L-1} e^{-\mathbf{j} 2 \pi n \Delta f \tau_{l}} \alpha_{i}(l),
$$

where $\alpha_{i}(l)=\left[\alpha_{1 i}(l), \cdots, \alpha_{M_{t} i}(l)\right]^{\mathcal{T}}$ denotes the channel gains from all transmit antennas to receive antenna $i$ for the $l$-th delay path. With the assumption that different delay paths are independent, which holds when different clusters of scatterers are well separated in the spatial domain, we have

$$
E\left[\mathbf{h}_{i}(n) \mathbf{h}_{i}^{\mathcal{H}}(k)\right]=\sum_{l=0}^{L-1} e^{-\mathbf{j} 2 \pi(n-k) \Delta f \tau_{l}} \mathbf{R}_{\alpha, l} .
$$

where the $M_{t} \times M_{t}$ matrix $\mathbf{R}_{\alpha, l}, 0 \leq l \leq L-1$, denotes the spatial fading covariance matrix of the $l$-th delay path to any receive antenna, and is given by

$$
\mathbf{R}_{\alpha, l}=E\left[\alpha_{j}(l) \alpha_{j}^{\mathcal{H}}(l)\right] .
$$

Combining (11) and (13), we can calculate $\Psi$ as shown in (15). With the fact that $\boldsymbol{\Delta}(n)$ is the $n$-th column of the matrix $(\mathbf{B}-\tilde{\mathbf{B}})$, we can further simplify (15) as follows

$$
\Psi=\sum_{l=0}^{L-1} \mathbf{D}^{\tau_{l}}(\mathbf{B}-\tilde{\mathbf{B}})^{T} \mathbf{R}_{\alpha, l}(\mathbf{B}-\tilde{\mathbf{B}})^{*} \mathbf{D}^{* \tau_{l}},
$$

where $\mathbf{D}=\operatorname{diag}\left(1, e^{-j 2 \pi \Delta f}, \ldots, e^{-j 2 \pi(N-1) \Delta f}\right)$.

Finally, we write (16) in a compact matrix form as follows

$$
\Psi=\mathbf{F} \operatorname{diag}\left[\mathbf{R}_{\alpha, 0}, \mathbf{R}_{\alpha, 1}, \cdots, \mathbf{R}_{\alpha, L-1}\right] \mathbf{F}^{\mathcal{H}},
$$

where $\mathbf{F}$ is given by

$$
\mathbf{F}=\left[\begin{array}{lll}
\mathbf{D}^{\tau_{0}}(\mathbf{B}-\tilde{\mathbf{B}})^{T} & \cdots & \mathbf{D}^{\tau_{L-1}}(\mathbf{B}-\tilde{\mathbf{B}})^{T}
\end{array}\right] .
$$

Therefore, combining (10) and (17), we get

$$
\mathbf{R}_{\boldsymbol{\Phi}}=\mathbf{I}_{M_{r}} \otimes \Psi
$$

We summarize the result of the above analysis in the following Theorem.

Theorem 1: The covariance matrix $\mathbf{R}_{\boldsymbol{\Phi}}$ can be decomposed as

$$
\mathbf{R}_{\boldsymbol{\Phi}}=\mathbf{I}_{M_{r}} \otimes \mathbf{F} \operatorname{diag}\left[\mathbf{R}_{\alpha, 0}, \mathbf{R}_{\alpha, 1}, \cdots, \mathbf{R}_{\alpha, L-1}\right] \mathbf{F}^{\mathcal{H}},
$$

where $\mathbf{R}_{\alpha, l}$ is specified in (14) and $\mathbf{F}$ is defined in (18).

Theorem 1 determines the covariance matrix $\mathbf{R}_{\boldsymbol{\Phi}}$ as a function of the spatial correlation structure of the channel and the SF-beamformer symbol B. This theorem will serve as a basis for the design of the beamformer.

\section{B. General Optimization Formulation for Beamformer Design}

We try to jointly design a general SF-beamformer matrix $\mathbf{B}$ that minimizes the system pairwise error probability (7) with the energy constraint $E\left[\|\mathbf{B}\|_{F}^{2}\right] \leq N M_{t}$. More specifically, the optimization problem can be stated as follows

$$
\begin{aligned}
& \min \left(\frac{\rho}{4 M_{t}}\right)^{-r\left(\mathbf{R}_{\boldsymbol{\Phi}}\right)}\left(\prod_{i=0}^{r\left(\mathbf{R}_{\boldsymbol{\Phi}}\right)-1} \mu_{i}\left(\mathbf{R}_{\boldsymbol{\Phi}}\right)^{-1}\right), \\
& \text { s.t. } E\left[\|\mathbf{B}\|_{F}^{2}\right] \leq N M_{t},
\end{aligned}
$$

where the minimization is over all possible SF-beamformer symbol pairs (minimize the worst case codewords pair) and the matrix $\mathbf{R}_{\Phi}$ is specified in (20). The objective function (21) suggests two criteria to design a general SF-beamformer symbol B: i) Maximize the rank of the matrix $\mathbf{R}_{\Phi}$; diversity gain; ii) maximize the product of the nonzero eigenvalues of $\mathbf{R}_{\boldsymbol{\Phi}}$ given by $\prod_{i=0}^{r\left(\mathbf{R}_{\Phi}\right)-1} \mu_{i}\left(\mathbf{R}_{\boldsymbol{\Phi}}\right)$; the coding gain. We emphasize at this point that the above design criteria for SFbeamformer are general in the sense that we do not impose any structure on the SF-beamformer B. We try to design a SF-beamformer matrix that matches to the channel statistical information available at the transmitter. If there is no spatial correlation at the transmitter side, i.e., the spatial correlation matrices $\mathbf{R}_{\alpha, l}$ are identity, the above conditions reduce to the design of SF codes [17].

The general optimization problem is difficult to tackle analytically. To relax the optimization problem, we will adopt another transmitting scheme in which a SF code is already designed to achieve full diversity for a spatial correlation-free channel, and then we try to design a beamformer to match to the channel correlation matrix. This approach of splitting the transmitter design problem into a predesigned SF code and a beamformer has been adopted in [8] for space-time coding over flat fading channels.

We denote the SF code by a $M_{t} \times N$ matrix $\mathbf{C}$. The linear transformation, or beamformer $\mathbf{W}$, can take various forms, for 


$$
\begin{aligned}
& \Psi=E\left\{\left[\begin{array}{c}
\boldsymbol{\Delta}^{T}(0) \mathbf{h}_{i}(0) \\
\vdots \\
\boldsymbol{\Delta}^{T}(N-1) \mathbf{h}_{i}(N-1)
\end{array}\right]\left[\mathbf{h}_{i}^{\mathcal{H}}(0) \boldsymbol{\Delta}^{*}(0), \ldots, \mathbf{h}_{i}^{\mathcal{H}}(N-1) \boldsymbol{\Delta}^{*}(N-1)\right]\right\} \\
& \Psi=\sum_{l=0}^{L-1} E\left\{\left[\begin{array}{c}
\boldsymbol{\Delta}^{T}(0) \alpha_{i}(l) \\
\vdots \\
e^{-\mathbf{j} 2 \pi(N-1) \Delta f \tau_{l}} \boldsymbol{\Delta}^{T}(N-1) \alpha_{i}(l)
\end{array}\right]\left[\begin{array}{lll}
\alpha_{i}^{\mathcal{H}}(l) \boldsymbol{\Delta}^{*}(0), & \ldots, & \alpha_{i}^{\mathcal{H}}(l) \boldsymbol{\Delta}^{*}(N-1) e^{\mathbf{j} 2 \pi(N-1) \Delta f \tau_{l}}
\end{array}\right]\right\}
\end{aligned}
$$

example: i) $\operatorname{vec}(\mathbf{B})=\mathbf{W} \operatorname{vec}(\mathbf{C})$ in which the beamformer matrix $\mathbf{W}$ is of size $N M_{t} \times N M_{t}$; ii) $\mathbf{B}=\mathbf{W C}$ in which the beamformer $\mathbf{W}$ is of size $M_{t} \times M_{t}$. In general, we represent the relation between the SF-beamformer symbol $\mathbf{B}$ and the SF-code $\mathbf{C}$ as follows

$$
\mathbf{f}(\mathbf{B})=\mathbf{W f}(\mathbf{C}),
$$

where $\mathbf{f}(\cdot)$ is a function that can, for example, take the form $\mathbf{f}(\mathbf{B})=\operatorname{vec}(\mathbf{B})$ or $\mathbf{f}(\mathbf{B})=\mathbf{B}$. Note that (22) is a general representation of all possible linear transformations between $\mathbf{C}$ and $\mathbf{B}$. Since we can think of $\mathbf{f}(\cdot)$ as a rearrangement of $\mathbf{B}$, we can write $\mathbf{B}$ as a product of a function of $\mathbf{W}$ and a function of $\mathbf{C}$ as follows

$$
\mathbf{B}=\mathbf{g}(\mathbf{W}) \mathbf{q}(\mathbf{C}),
$$

where $\mathbf{g}(\cdot)$ is an $M_{t} \times K$ matrix, $\mathbf{q}(\cdot)$ is a $K \times N$ matrix, and $K$ depends on the function $\mathbf{f}(\cdot)$. Substituting (23) into (20), we get (24), where $\hat{\mathbf{F}}=$ $\left[\begin{array}{lll}\mathbf{D}^{\tau_{0}}(\mathbf{q}(\mathbf{C})-\mathbf{q}(\tilde{\mathbf{C}}))^{T} & \cdots & \mathbf{D}^{\tau_{L-1}}(\mathbf{q}(\mathbf{C})-\mathbf{q}(\tilde{\mathbf{C}}))^{T}\end{array}\right]$.

In order to apply the design criteria we need to find the rank and eigenvalues of the matrix $\mathbf{R}_{\boldsymbol{\Phi}}$ in terms of the beamformer matrix $\mathbf{W}$. In order to simplify the notations, let the $L K \times L K$ matrix $\tilde{\mathbf{R}}$ denote the block diagonal matrix in (24) as shown in (25). Then, the rank of $\mathbf{R}_{\boldsymbol{\Phi}}$ can be given by $r\left(\mathbf{R}_{\Phi}\right)=M_{r} r\left(\hat{\mathbf{F}} \tilde{\mathbf{R}} \hat{\mathbf{F}}^{\mathcal{H}}\right)$. The matrix $\hat{\mathbf{F}}$ is of size $N \times L K$, and we assume that $L K \leq N$, which is typically true in OFDM systems as $N$ is usually designed much larger than $L M_{t}$. Assuming that the SF code is designed to achieve full diversity in the case of no spatial correlation, we rewrite $\hat{\mathbf{F}} \tilde{\mathbf{R}} \hat{\mathbf{F}}^{\mathcal{H}}$ after row and column reordering in the form

$$
\mathbf{J}=\left[\begin{array}{c}
\hat{\mathbf{F}}_{1} \\
\hat{\mathbf{F}}_{2}
\end{array}\right] \tilde{\mathbf{R}}\left[\begin{array}{ll}
\hat{\mathbf{F}}_{1}^{\mathcal{H}} & \hat{\mathbf{F}}_{2}^{\mathcal{H}}
\end{array}\right]=\left[\begin{array}{cc}
\hat{\mathbf{F}}_{1} \tilde{\mathbf{R}} \hat{\mathbf{F}}_{1}^{\mathcal{H}} & \hat{\mathbf{F}}_{1} \tilde{\mathbf{R}} \hat{\mathbf{F}}_{2}^{\mathcal{H}} \\
\hat{\mathbf{F}}_{2} \tilde{\mathbf{R}} \hat{\mathbf{F}}_{1}^{\mathcal{H}} & \hat{\mathbf{F}}_{2} \tilde{\mathbf{R}} \hat{\mathbf{F}}_{2}^{\mathcal{H}}
\end{array}\right],
$$

where $\mathbf{J}$ is the reordered matrix, $\hat{\mathbf{F}}_{\mathbf{1}}$ is of size $L K \times L K$ and is full rank, and the matrix $\hat{\mathbf{F}}_{2}$ takes the rest of the matrix. Since the ordered singular values of a matrix are not smaller than the corresponding singular values of any square submatrix obtained by deleting equal number of rows and columns of the original matrix [19], we get

$$
\mu_{i}\left(\hat{\mathbf{F}} \tilde{\mathbf{R}} \hat{\mathbf{F}}^{\mathcal{H}}\right) \geq \mu_{i}\left(\hat{\mathbf{F}}_{\mathbf{1}} \tilde{\mathbf{R}} \hat{\mathbf{F}}_{\mathbf{1}}^{\mathcal{H}}\right),
$$

where $\mu_{i}()$ denotes the $i$-th eigenvalue of a matrix and are ordered in non-increasing order.

The eigenvalues of $\hat{\mathbf{F}}_{\mathbf{1}} \tilde{\mathbf{R}} \hat{\mathbf{F}}_{\mathbf{1}}^{\mathcal{H}}$ are given by $\mu_{i}\left(\hat{\mathbf{F}}_{\mathbf{1}} \tilde{\mathbf{R}} \hat{\mathbf{F}}_{\mathbf{1}}^{\mathcal{H}}\right)=$ $\theta_{i} \mu_{i}(\tilde{\mathbf{R}})$, where $\theta_{i}$ is a nonnegative real number such that $\mu_{\min }\left(\hat{\mathbf{F}}_{\mathbf{1}} \hat{\mathbf{F}}_{\mathbf{1}}^{\mathcal{H}}\right) \leq \theta_{i} \leq \mu_{\max }\left(\hat{\mathbf{F}}_{\mathbf{1}} \hat{\mathbf{F}}_{\mathbf{1}}^{\mathcal{H}}\right)$, which follows by
Ostrowski [20], and $\mu_{\min }$ and $\mu_{\max }$ denote the smallest and largest eigenvalues, respectively. Applying Ostrowski's Theorem along with (27), we can find the rank of the matrix $\hat{\mathbf{F}} \tilde{\mathbf{R}} \hat{\mathbf{F}}^{\mathcal{H}}$ as follows

$$
r\left(\hat{\mathbf{F}} \tilde{\mathbf{R}} \hat{\mathbf{F}}^{\mathcal{H}}\right)=r(\tilde{\mathbf{R}})=\sum_{l=0}^{L-1} r\left(\mathbf{g}^{T}(\mathbf{W}) \mathbf{R}_{\alpha, l} \mathbf{g}^{*}(\mathbf{W})\right),
$$

where the second equality comes from the block diagonal structure of $\tilde{\mathbf{R}}$. Similarly, the eigenvalues of the matrix $\hat{\mathbf{F}} \tilde{\mathbf{R}} \hat{\mathbf{F}}^{\mathcal{H}}$ can be lower bounded as follows

$$
\mu_{i}\left(\hat{\mathbf{F}} \tilde{\mathbf{R}} \hat{\mathbf{F}}^{\mathcal{H}}\right) \geq \mu_{\min }\left(\hat{\mathbf{F}}_{\mathbf{1}} \hat{\mathbf{F}}_{\mathbf{1}}^{\mathcal{H}}\right) \mu_{i}(\tilde{\mathbf{R}}) .
$$

Note that, maximizing the coding gain of the system corresponds to maximizing the product of the nonzero eigenvalues of the matrix $\mathbf{R}_{\boldsymbol{\Phi}}$ which is equivalent to maximizing the product of the nonzero eigenvalues of $\hat{\mathbf{F}} \tilde{\mathbf{R}} \hat{\mathbf{F}}^{\mathcal{H}}$. From (29), this product can be lower bounded as follows

$$
\prod_{i=1}^{r(\tilde{\mathbf{R}})} \mu_{i}\left(\hat{\mathbf{F}} \tilde{\mathbf{R}} \hat{\mathbf{F}}^{\mathcal{H}}\right) \geq \gamma \prod_{i=1}^{r(\tilde{\mathbf{R}})} \mu_{i}(\tilde{\mathbf{R}}) .
$$

where $\gamma$ is a constant that depends on $\mu_{\min }\left(\hat{\mathbf{F}}_{\mathbf{1}} \hat{\mathbf{F}}_{\mathbf{1}}^{\mathcal{H}}\right)$. If the matrix $\tilde{\mathbf{R}}$ is full rank, the product of its eigenvalues corresponds to its determinant. Thus our goal now is to maximize the determinant of the matrix $\tilde{\mathbf{R}}$ under the energy constraint on the SF-beamformer symbol. The determinant of the matrix $\tilde{\mathbf{R}}$ is upper bounded by the product of its diagonal elements [20], and the upper-bound is achieved if and only if $\tilde{\mathbf{R}}$ is diagonal. More specifically

$$
\operatorname{det}(\tilde{\mathbf{R}}) \leq \prod_{i=1}^{L K} \tilde{\mathbf{R}}_{i i}
$$

where $\tilde{\mathbf{R}}_{i i}$ is the $i$-th diagonal element of the matrix $\tilde{\mathbf{R}}$. Hence, the equality holds when the matrix $\tilde{\mathbf{R}}$ is diagonalized and this can only be achieved if the $L$ block diagonal entries of the matrix $\tilde{\mathbf{R}}$ are diagonalized (25). This corresponds to choosing $\mathbf{W}$ to diagonalize $\mathbf{g}^{T}(\mathbf{W}) \mathbf{R}_{\alpha, l} \mathbf{g}^{*}(\mathbf{W})$, for all $0 \leq l \leq(L-1)$.

A beamformer that achieves the upper bound in (31) is considered optimal. However, according to (24), irrespective of the form of the function $\mathbf{g}(\mathbf{W})$ the same beamformer should match to the covariance matrices of all the $L$ delay paths simultaneously in order to achieve the upper bound. This can not be achieved, in general, except for the special cases when all of the $L$ delay paths have the same spatial correlation matrix, or when $L=1$ which corresponds to the flat fading case. As a result, it is very difficult, if not impossible, to find a 


$$
\begin{gathered}
\mathbf{R}_{\boldsymbol{\Phi}}=\mathbf{I}_{M_{r}} \otimes \hat{\mathbf{F}} \operatorname{diag}\left[\mathbf{g}^{T}(\mathbf{W}) \mathbf{R}_{\alpha, 0} \mathbf{g}^{*}(\mathbf{W}), \mathbf{g}^{T}(\mathbf{W}) \mathbf{R}_{\alpha, 1} \mathbf{g}^{*}(\mathbf{W}), \cdots, \mathbf{g}^{T}(\mathbf{W}) \mathbf{R}_{\alpha, L-1} \mathbf{g}^{*}(\mathbf{W})\right] \hat{\mathbf{F}}^{\mathcal{H}}, \\
\tilde{\mathbf{R}}=\operatorname{diag}\left[\mathbf{g}^{T}(\mathbf{W}) \mathbf{R}_{\alpha, 0} \mathbf{g}^{*}(\mathbf{W}), \mathbf{g}^{T}(\mathbf{W}) \mathbf{R}_{\alpha, 1} \mathbf{g}^{*}(\mathbf{W}), \cdots, \mathbf{g}^{T}(\mathbf{W}) \mathbf{R}_{\alpha, L-1} \mathbf{g}^{*}(\mathbf{W})\right]
\end{gathered}
$$

closed form solution for the optimal beamformer. To solve the optimization problem we must, in general, employ numerical search techniques for the beamformer matrix, which will be exhaustive. The above optimization problem is challenging, as it is different from the problem of beamforming in a MIMO flat fading channel in the sense that the transmitter should beamform across multiple eigenspaces simultaneously. To provide some insights we will render to suboptimal solutions for the problem, and for simplicity, we will adopt the conventional definition of the beamformer $\mathbf{B}=\mathbf{W C}$ in the rest of the paper.

\section{Suboptimal Designs of Beamformers}

In this section, three different approaches for designing the beamformer are proposed. The proposed approaches, although suboptimal, are well motivated by the derived performance criteria and the understanding of the underlying physics of the problem.

\section{A. Eigenvalue Selection Scheme}

In this subsection, we design the beamformer jointly for all subcarriers, and propose the Eigenvalue selection scheme. The optimization problem can be re-casted in the following manner. There are $\mathrm{L}$ independent delay paths, each has $M_{t}$ degrees of freedom. The beamformer has only $M_{t}$ degrees of freedom to match to the $L M_{t}$-dimensional spaces shown in (32). We represent the beamformer $\mathbf{W}$ in the following way

$$
\mathbf{W}=\mathbf{U} \Gamma
$$

where the $i$-th column in $\mathbf{U}$ corresponds to the $i$-th beamforming direction, and $\Gamma$ is a diagonal matrix with the $i$-th diagonal element representing the power loading along this direction. The beamformer directions $\mathbf{U}$ should be designed to simultaneously whiten the effects of the spatial correlation matrices $\left\{\mathbf{R}_{\alpha, l}\right\}_{l=0}^{L-1}$ in (32) in order to satisfy the upper bound in (31), which is very general to achieve in general.

One intuitive, but suboptimal, approach to overcome this problem is to select from amongst the $L M_{t}$-dimensional space an $M_{t}$ space and design the beamformer to match this smaller space. One can choose this smaller space according to different criteria. In the Eigenvalue selection approach, we choose the largest $M_{t}$ eigenvalues $\lambda_{i}$ from the $L M_{t}$ eigenvalues available from the eigendecomposition of the $L$ covariance matrices, and the corresponding $M_{t}$ eigenvectors $\mathbf{v}_{i}$. The beamformer is then designed to transmit in the directions of these eigenvectors and the power loading is done proportional to the eigenvalues along these directions. The rationale behind doing the power loading in this way is that in general the available power should be distributed according to the channel conditions, i.e., more power should be allocated to channels with better quality. Simulation results show that this approach works fairly well under different channel conditions. The algorithm can be summarized in the following steps:
1) Let the eigendecomposition of the spatial correlation matrix at the $l$-th path be given by $\mathbf{R}_{\alpha, l}=\mathbf{V}_{l} \Lambda_{l} \mathbf{V}_{l}^{\mathcal{H}}$, where $0 \leq l \leq L-1$.

2) Choose the largest $M_{t}$ eigenvalues and the corresponding eigenvectors from the $L M_{t}$ available eigenvalues and eigenvectors in $\Lambda_{l}$ and $\mathbf{V}_{l}, l=0,1, \cdots, L-1$.

3) Arrange the $M_{t}$ selected pairs in matrix format as follows $\Lambda=\operatorname{diag}\left(\lambda_{1}, \cdots, \lambda_{M_{t}}\right), \mathbf{V}=\left[\mathbf{v}_{1}, \cdots, \mathbf{v}_{M_{t}}\right]$.

4) The beamformer $\mathbf{W}$ is determined as $\mathbf{W}=\mathbf{U} \Gamma$, in which $\mathbf{U}=\mathbf{V}^{*}, \Gamma=\operatorname{diag}\left(\sigma_{1}, \cdots, \sigma_{M_{t}}\right)$, and $\sigma_{i}^{2}=\frac{\lambda_{i}}{\sum_{l=0}^{L-1} \lambda_{i}}$.

The SF-beamformer symbol $\mathbf{B}$ is required to satisfy the energy constraint $E\left[\|\mathbf{B}\|_{F}^{2}\right]=N M_{t}$ as stated before, then we can normalize the resultant SF-beamformer symbol to satisfy $\mathbf{B}=$ $\frac{\mathrm{WC}}{\|\mathrm{WC}\|_{F}} \sqrt{N M_{t}}$, which guarantees that the energy of the SFbeamformer symbol B does not exceed $N M_{t}$.

It can be expected that choosing the directions with the largest eigenvalues, i.e., with the most reliable channel conditions, enhances the coding gain. However, since the directions associated with the beamformer belong to different eigenspaces (they belong to different eigendecompositions), they are no more orthogonal and the matrix $\mathbf{V}$ is not, in general, full rank. Accordingly, the matrix $\mathbf{U}$ is not, in general, full rank and hence full diversity is not guaranteed in the Eigenvalue selection approach. We will explore this more in another approach described next.

\section{B. Eigenspace Selection Scheme}

In this scheme, we try to jointly select the eigenvalues and eigenvectors, not only based on coding gain, but also based on the diversity order of the system. The criteria that we suggest is maximizing the volume occupied by the beamformer matrix, which is given by the absolute value of the determinant of the beamformer matrix

$$
\mathbf{W}=\underset{\substack{\lambda_{i}, \mathbf{v}_{i} \\ i=1, \cdots, L M_{t}}}{\operatorname{argmax}}|\operatorname{det}(\mathbf{W})| .
$$

To understand the intuition behind using this cost function, let us investigate the $M_{t}=2$ case, in which the beamformer can be written as follows $\mathbf{W}=\left[\mathbf{u}_{1} \mathbf{u}_{2}\right] \operatorname{diag}\left[\sigma_{1}, \sigma_{2}\right]$. In this case, the criteria is proportional to the area spanned by the matrix $\mathbf{W}$. This area is given by $\sigma_{1} \sigma_{2} \sin \left(<\mathbf{u}_{1}, \mathbf{u}_{2}>\right)$, where $\mathbf{u}_{i}$ and $\sigma_{i}$ are the $i$-th eigenbeam and associated allocated power respectively, and $\langle\cdot, \cdot\rangle$ denotes the angle between the two vectors. Clearly, the coding gain is controlled by the part $\sigma_{1} \sigma_{2}$, which corresponds to the power loading and the magnitude of the channel eigenvalues. The diversity gain is controlled by $\left.\sin \left(<\mathbf{u}_{1}, \mathbf{u}_{2}\right\rangle\right)$. Note that full diversity corresponds to the case when the two eigenvectors $\mathbf{u}_{1}$ and $\mathbf{u}_{2}$ are orthogonal, while diversity order one results when these two vectors are parallel.

In a higher dimensional space, the volume occupied by the beamformer, given by $|\operatorname{det}(\mathbf{W})|$, is the volume spanned 


$$
\mathbf{R}_{\boldsymbol{\Phi}}=\mathbf{I}_{M_{r}} \otimes \hat{\mathbf{F}} \operatorname{diag}\left[\mathbf{W}^{T} \mathbf{R}_{\alpha, 0} \mathbf{W}^{*}, \mathbf{W}^{T} \mathbf{R}_{\alpha, 1} \mathbf{W}^{*}, \cdots, \mathbf{W}^{T} \mathbf{R}_{\alpha, L-1} \mathbf{W}^{*}\right] \hat{\mathbf{F}}^{\mathcal{H}}
$$

by a parallelepiped in an $M_{t}$-dimensional space, which is determined by the lengths of its sides and the angles between these sides. The length of a side correspond to the magnitude of the eigenvalue associated with this side, while the angles between the sides affect the diversity gain. Full diversity is achieved if all sides are orthogonal. Hence maximizing $\operatorname{det}(\mathbf{W})$ provides a tradeoff between the coding gain and the diversity order achieved by the system, and it would be expected that such a scheme would provide a performance tradeoff between pure SF coding that achieves full diversity and the Eigenvalue selection scheme that maximizes the coding gain of the system. We summarize the algorithm for the Eigenspace selection scheme in the following steps:

1) Let the eigendecomposition of the spatial correlation matrix at the $l$-th path be given by $\mathbf{R}_{\alpha, l}=\mathbf{V}_{l} \Lambda_{l} \mathbf{V}_{l}^{\mathcal{H}}$, where $0 \leq l \leq L-1$.

2) Choose every possible combination of $M_{t}$ eigenvalue and eigenvector pairs from the $L M_{t}$ pairs available from the eigendecomposition in the previous step.

3) Arrange the $M_{t}$ selected pairs in matrix format as follows $\Lambda=\operatorname{diag}\left(\lambda_{1}, \cdots, \lambda_{M_{t}}\right), \mathbf{V}=\left[\mathbf{v}_{1}, \cdots, \mathbf{v}_{M_{t}}\right]$.

4) The beamformer $\mathbf{W}$ is determined as $\mathbf{W}=\mathbf{U} \Gamma$, in which $\mathbf{U}=\mathbf{V}^{*}, \Gamma=\operatorname{diag}\left(\sigma_{1}, \cdots, \sigma_{M_{t}}\right)$, and $\sigma_{i}^{2}=\frac{\lambda_{i}}{\sum_{l=0}^{L-1} \lambda_{i}}$.

5) Calculate $|\operatorname{det}(\mathbf{W})|$.

6) From among all possible combinations, choose $\mathbf{W}$ with the largest determinant.

Similar to the Eigenvalue selection scheme, the columns of the matrix $\mathbf{U}$ in the Eigenspace selection algorithm are not, in general, orthogonal as the directions of the beamformer belong to different eigenspaces. Hence, we need to normalize the energy of the new SF-beamformer symbol as $\mathbf{B}=\frac{\mathbf{W C}}{\|\mathbf{W C}\|} \sqrt{N M_{t}}$.

\section{Per-subcarrier Solution}

In the previous two approaches, the beamformer was designed for all the subcarriers. In the per-subcarrier approach, we try to design a beamformer to whiten the spatial correlation effect of the channel at each subcarrier.

Assuming ML detection over each subcarrier, the average pairwise error probability at the $n$-th subcarrier between two channel symbol vectors $\mathbf{b}(n)$ and $\tilde{\mathbf{b}}(n)$ can be upper bounded by

$$
\begin{aligned}
& \operatorname{Pr}(\mathbf{b}(n) \rightarrow \tilde{\mathbf{b}}(n)) \\
& \quad \leq\left(\frac{\rho}{4 M_{t}}\right)^{-r\left(\mathbf{R}_{\Phi}(n)\right)}\left(\prod_{i=0}^{r\left(\mathbf{R}_{\Phi}(n)\right)-1} \mu_{i}\left(\mathbf{R}_{\Phi}(n)\right)^{-1}\right),
\end{aligned}
$$

where $\mathbf{R}_{\boldsymbol{\Phi}}(n)$ can be shown to be given by (36). According to the spatial correlation model specified in (2) and the assumption of independence between different delays, we have

$$
E\left[\mathbf{h}_{i}(n) \mathbf{h}_{j}^{\mathcal{H}}(n)\right]=\delta(i-j) \sum_{l=0}^{L-1} \mathbf{R}_{\alpha, l},
$$

which is the same for all receive antennas. Substituting (37) into (36), we get (38). Equation (38) asserts that independent of the subcarrier, the beamformer should match to the same matrix which is given by $\sum_{l=0}^{L-1} \mathbf{R}_{\alpha, l}$. As a result, for this approach, the transmitter can apply the same beamformer to all of the transmitted symbols. The interpretation for this result is that the channel transfer function at any subcarrier is given by the FFT of the channel gains at all delays, and thus they all have the same spatial information about the channel. The algorithm works as follows.

- Let the eigendecomposition of the matrix $\sum_{l=0}^{L-1} \mathbf{R}_{\alpha, l}$ be given by $\sum_{l=0}^{L-1} \mathbf{R}_{\alpha, l}=\mathbf{V} \Lambda \mathbf{V}^{\mathcal{H}}$.

- The beamformer directions $\mathbf{U}$ are chosen to whiten the effect of the matrix $\sum_{l=0}^{L-1} \mathbf{R}_{\alpha, l}$ as $\mathbf{U}=\mathbf{V}^{*}$.

- The power loading across the beamformer directions is done similar to the Eigenvalue and Eigenspace approaches as $\sigma_{i}^{2}=\frac{\lambda_{i}}{\sum_{l=0}^{L-1} \lambda_{i}}$.

\section{Simulation Results}

To demonstrate the performance improvement due to applying the proposed algorithms compared to that of SF coding without beamforming, we performed some computer simulations. The channel model used is a two-ray, equal-power delay profile, with a delay of $20 \mu \mathrm{s}$ between the two rays. The MIMO-OFDM system has $N=128$ subcarriers, and QPSK modulation is used. The total bandwidth of the system is $1 M H z$. Two antennas are used at the transmitter side, and one at the receiver. ${ }^{1}$

We choose the full-diversity SF code design from [17] to conduct the simulations. Essentially, the SF code is obtained by mapping from ST codes. In our simulations we use the $2 \times 2$ Alamouti's code [2]. To generate the spatial correlation channel coefficients, we use the following model $\alpha_{l}=\mathbf{A}_{l} \tilde{\alpha}_{l}$, where $l \in\{0, \cdots, L-1\}, \alpha_{l}=$ $\left[\begin{array}{ccc}\alpha_{1}^{T}(l), & \cdots, & \alpha_{M_{r}}^{T}(l)\end{array}\right]^{\mathcal{T}}, \tilde{\alpha}_{l}$ is an $M_{r} M_{t} \times 1$ vector with i.i.d entries chosen from a complex Gaussian distribution with zero mean and variance $\beta_{l}^{2}$, and the matrix $\mathbf{A}_{l}$ contains the correlation coefficients, as follows

$$
\mathbf{A}_{l} \mathbf{A}_{l}^{\mathcal{H}}=\mathbf{I}_{M_{r}} \otimes \mathbf{R}_{\alpha, l} .
$$

Three channel scenarios are considered in the simulation experiments: i) Channel 1: The eigenvalues of the $2 \times 2$ matrix $\mathbf{R}_{\alpha, l}, l \in\{0,1\}$, has one non-zero eigenvalue, i.e., the matrix has rank one. This can be considered as a highly correlated scenario. ii) Channel 2: The eigenvalues for $\mathbf{R}_{\alpha, 0}$ are 0.22 and 0.77 , and for $\mathbf{R}_{\alpha, 1}$ are 1.4 and .075 . This corresponds to a moderate correlated channel model, and actually from random experiments we find that most of the channel realizations behave in this way. iii) Channel 3: The eigenvalues for $\mathbf{R}_{\alpha, 0}$ are 0.13 and 0.8 , and for $\mathbf{R}_{\alpha, 1}$ are 0.7 and 0.2 . This corresponds to a channel with low spatial correlation, as the values of the eigenvalues are comparable.

\footnotetext{
${ }^{1}$ In the simulations, we did not consider exhaustive search for the optimal global solution of the beamformer because the computations are prohibitive.
} 


$$
\begin{aligned}
& \mathbf{R}_{\boldsymbol{\Phi}}(n)=E\left\{\left[\begin{array}{c}
\boldsymbol{\Delta}^{T}(n) \mathbf{h}_{\mathbf{1}}(n) \\
\vdots \\
\boldsymbol{\Delta}^{T}(n) \mathbf{h}_{M_{r}}(n)
\end{array}\right]\left[\mathbf{h}_{1}^{\mathcal{H}}(n) \boldsymbol{\Delta}^{*}(n), \cdots, \mathbf{h}_{M_{r}}^{\mathcal{H}}(n) \boldsymbol{\Delta}^{*}(n)\right]\right\} \\
& \mathbf{R}_{\boldsymbol{\Phi}}(n)=\left[(\mathbf{c}(n)-\tilde{\mathbf{c}}(n))^{T} \mathbf{W}^{T}(n)\left(\sum_{l=0}^{L-1} \mathbf{R}_{\alpha, l}\right) \mathbf{W}^{*}(n)(\mathbf{c}(n)-\tilde{\mathbf{c}}(n))^{*}\right] \mathbf{I}_{M_{r}} .
\end{aligned}
$$

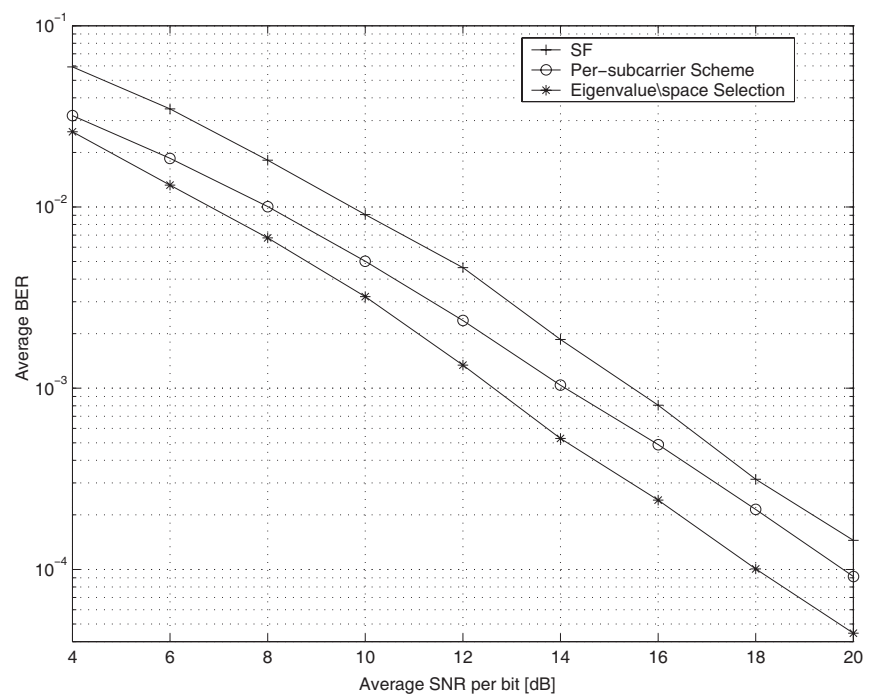

Fig. 1. Bit error rate performance comparison: Channel 1 with $M_{t}=2$ transmit and $M_{r}=1$ receive antennas.

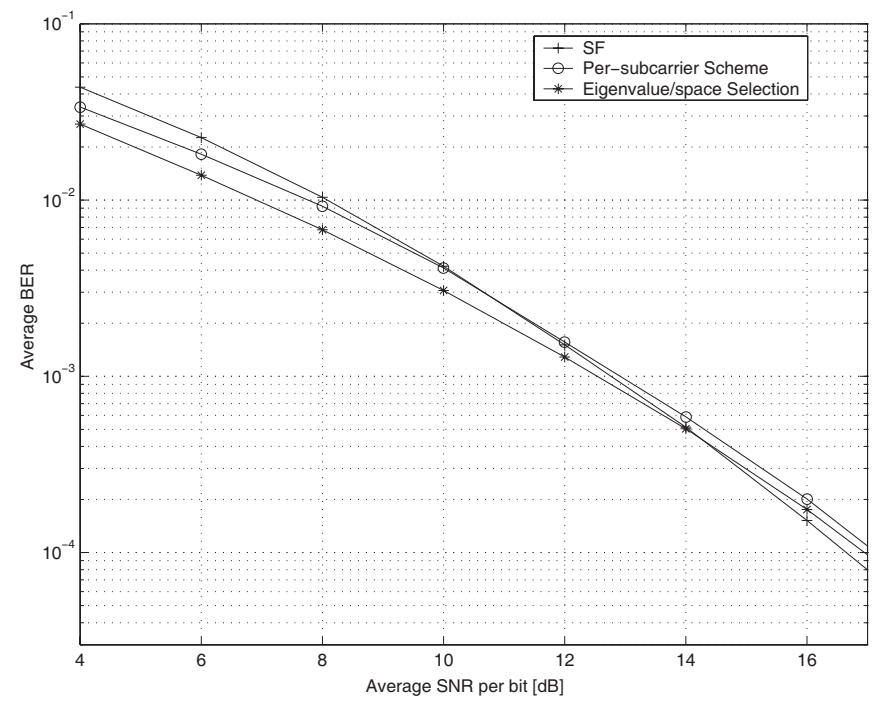

Fig. 2. Bit error rate performance comparison: Channel 2 with $M_{t}=2$ transmit and $M_{r}=1$ receive antennas.

Fig. 1 depicts the results for Channel 1. Both the Eigenvalue and Eigenspace selection schemes choose the same eigenvector pair, as this channel has only one non-zero eigenvalue. As shown in the results, the proposed algorithms have better performance compared to that of SF coding without beamforming. Also, it can be seen that the performance curves are approximately parallel, as the beamformer achieves codinggain, and does not incur any diversity loss in this case due to

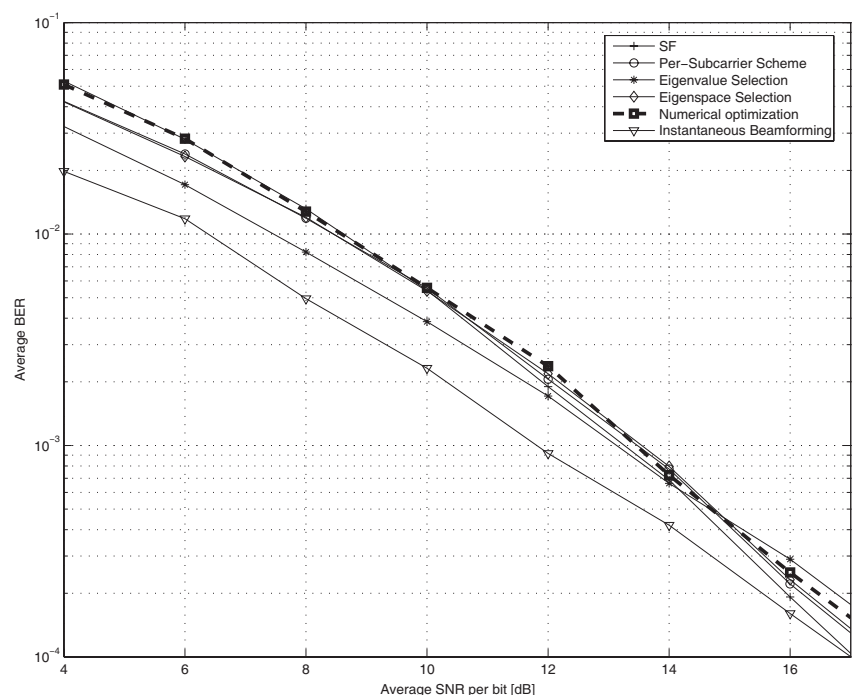

Fig. 3. Bit error rate performance comparison: Channel 3 with $M_{t}=2$ transmit and $M_{r}=1$ receive antennas. The dashed line represent the performance results when using numerical optimization to design the beamformer.

the fact that Channel 1 is highly correlated. The performance of the Eigenvalue selection scheme is better than that of the per-subcarrier algorithm, and it has approximately $3 \mathrm{~dB}$ gain over SF coding without beamforming. This is due to the fact that the Eigenvalue selection algorithm beamforms in the two non-zero directions, while SF coding distributes its power equally among all directions, hence losing half its power.

Second, we consider Channel 2 as shown in Fig. 2. Similar to Channel 1, both the Eigenvalue and Eigenspace selection schemes choose the same eigenvector pair, and thus have the same performance. The results show that the Eigenvalue selection scheme gives the best performance in low to medium SNR regions, while it is inferior to SF coding at sufficiently high SNR regions. This can be interpreted as follows: Since Channel 2 is less correlated than Channel 1, the eigenvalues are more spread. In the region of low and medium SNR, sending information on the most reliable channels gives the best performance, this is achieved by the Eigenvalue selection scheme as it chooses the directions with largest eigenvalues. While in high SNR region, diversity gain dominates the performance, which corresponds to equal power loading along all directions, and it is achieved by SF coding.

Finally, we consider Channel 3. In this scenario, Eigenvalue and Eigenspace beamforming choose different eigenvector pairs. From Fig. 3, at low to medium SNR, Eigenvalue selection still gives the best performance, while the performance becomes in favor of SF coding at high SNR regions. 
As expected, Eigenspace selection beamforming provides the tradeoff between the two extreme cases: Eigenvalue selection scheme, which corresponds to optimizing the coding gain, and SF coding without beamforming which achieves full diversity gain. Thus, at low to moderate SNR regions the performance of the Eigenspace algorithm is better than SF coding and inferior to Eigenvalue selection, while it is better than Eigenvalue selection and inferior to SF coding at high SNR regions. In Fig. 3, we have considered two more scenarios in our comparisons. In particular, we applied numerical optimization techniques to find the optimal beamforming design that satisfies (31). Since the problem is highly non-convex, the numerical algorithm is not guaranteed to converge to a global optimal, and only local optima are reached. The local optima depend heavily on the initial conditions selected and can lead to performance inferior to the proposed algorithms.

Since comparing our heuristic to the optimal solution is not feasible because the optimal solution can not be found in our case, we compared the performance of our algorithms to the best scenario possible when perfect instantaneous channel information is available at the transmitter. In this case, it was shown in [21], for the flat fading scenario, that transmitting along the eigenvector with the largest eigenvalue minimizes the error probability. We follow the same approach by designing a beamformer separately at each subcarrier. The results are depicted in Fig. 3 and it is clear that the gap between this best scenario and our heuristics is not large.

From the simulations we have the following observations. In the per-subcarrier approach, the beamformer $\mathbf{W}$ should match the sum of the spatial correlation matrices $\sum_{l=0}^{L-1} \mathbf{R}_{\alpha, l}$. This means that even if all or some of the spacial correlation matrices $R_{\alpha, l}$ are rank deficient, the resultant matrix need not be rank deficient, and this will affect the power loading part in the per-subcarrier approach, and results in inferior performance compared to Eigenvalue or Eigenspace selection especially at low SNR. This is clear for Channels 1 and 2 , where there is a relatively high spatial correlation. On the other hand, Channel 3 has relatively low correlation and that is why we can see that both the Eigenspace selection and the per-subcarrier will have very close performance to space-frequency coding without a beamformer as the resulting power loading across the beams will almost be uniform. This is not the case, however, with the Eigenvalue scheme which finds the largest eignvalues in all the spatial correlation matrices eigenspaces and beamforms in the their direction, which results in a better performance at low SNR regimes and worse performance at high SNR regimes.

\section{CONCLUSION}

We formulated a general optimization problem to jointly design a SF-beamformer scheme that minimizes the pairwise error probability of MIMO-OFDM systems with spatial correlation feedback. We derived the performance analysis for a MIMO-OFDM system with arbitrary spatial correlation, and provided the criteria to jointly design an optimum SFbeamformer at the transmitter. The analysis revealed that finding a closed form for the optimal beamformer design is not tractable due to the fact that the beamformer need to match to multiple eigenspaces simultaneously. Based on our analytical results, we proposed three suboptimal transmitting schemes. For any SF code designed for a spatially uncorrelated channel, we designed beamformers that match to the spatial covariance structure of the channel. Simulation results showed that the Eigenvalue selection scheme can achieve a $3 \mathrm{~dB}$ gain over $\mathrm{SF}$ coding without beamforming in highly correlated channels. For channels with lower correlations, it results in a better performance in low to moderate SNR regions.

\section{REFERENCES}

[1] V. Tarokh, N. Seshadri, and A. R. Calderbank, "Space-time codes for high data rate wireless communications: performance criterion and code construction," IEEE Trans. Inform. Theory, vol. 44, no. 2, pp. 744-765, Mar. 1998.

[2] S. Alamouti, "A simple transmit diversity technique for wireless communications," IEEE J. Select. Areas Commun., vol. 16, no. 8, pp. 14511458, 1998.

[3] F. Rashid-Farrokhi, K. J. R. Liu, and L. Tassiulas, "Transmit beamforming and power control for cellular wireless systems," IEEE J. Select. Areas Commun., vol. 16, pp. 1437-1450, Oct. 1998.

[4] D. P. Palomar, J. M. Cioffi, and M. A. Lagunas, "Joint Tx-Rx beamforming design for multicarrier MIMO channels: A unified framework for convex optimiztion," IEEE Trans. Signal Processing, vol. 51, pp. 2381-2401, Sept. 2003.

[5] A. Narula, M. J. Lopez, M. D. Trott, and G. W. Wornell, "Efficient use of side information in multiple-antenna data transmission over fading channels," IEEE J. Select. Areas Commun., vol. 16, pp. 1423-1436, Oct. 1998.

[6] E. Visotsky and U. Madhow, "Space-time transmit precoding with imperfect feedback," IEEE Trans. Inform. Theory, vol. 47, pp. 26322639, Sept. 2001.

[7] S. A. Jafar and A. Goldsmith, "On optimality of beamforming for multiple antenna systems with imperfect feedback," in Proc. Int. Symp. Information Theory, June 2001, p. 321.

[8] G. Jöngren, M. Skoglund, and B. Ottersten, "Combining beamforming and orthogonal space-time block coding," IEEE Trans. Inform. Theory, vol. 48, pp. 611-627, Mar. 2002.

[9] P. Xia, S. Zhou, and G. B. Giannakis, "Adaptive MIMO-OFDM based on partial channel state information," IEEE Trans. Signal Processing, vol. 52, pp. 202-213, Jan. 2004.

[10] A. K. Sadek, Weifeng Su, and K. J. R. Liu, "Eigen-selection approach for joint beamforming and space-frequency coding in MIMO-OFDM Systems with spatial correlation feedback," in Proc. IEEE Int. Workshop on Signal Processing Advances for Wireless Communications (SPAWC), pp. 588-592, New York, NY, June 2005.

[11] H. Bölcskei and A. J. Paulraj, "Space-frequency coded broadband OFDM systems," in Proc. IEEE WCNC, pp. 1-6, Sept. 2000.

[12] J. Choi and R. W. Heath, Jr. "Interpolation based transmit beamforming for MIMO-OFDM with limited feedback," in Proc. IEEE Int. Conf. on Communications, vol. 1, pp. 249-253, Paris, France, June 2004.

[13] J. Choi, S. R. Kim, and I.K. Choi "Eigenbeamforming with selection diversity for MIMO-OFDM downlink," in Proc. IEEE Vehicular Technology Conference, pp. 1806-1810, vol. 3, Sept. 2004.

[14] G. Barriac and U. Madhow "Space-time communication for OFDM with implicit channel feedback," IEEE Trans. Inform. Theory, vol. 50, no. 12, pp. 3111-3129, Dec. 2004.

[15] L. J. Cimini, "Analysis and simulation of a digital mobile channel using orthogonal frequency division multiplexing," IEEE Trans. Commun., vol. 33, pp. 665-675, July 1985.

[16] Y. Li, J. H. Winters, and N. R. Sollenberger, "MIMO-OFDM for wireless communications: signal detection with enhanced channel estimation," IEEE Trans. Commun., vol. 50, pp. 1471-1477, Sept. 2002.

[17] W. Su, Z. Safar, M. Olfat, and K. J. R. Liu, "Obtaining full-diversity space-frequency codes from space-time code via mapping," IEEE Trans. Signal Processing, vol. 51, no. 11, pp. 2905-2916, Nov. 2003.

[18] A. M. Mathai and S. B. Provost, Quadratic Forms in Random Variables. New York: Marcel Dekker, 1992.

[19] R. A. Horn and C. R. Johnson, Topics in Matrix Analysis. Cambridge Univ. Press, 1991.

[20] R. A. Horn and C. R. Johnson, Matrix Analysis. Cambridge Univ. Press, 1985.

[21] K. K. Mukkavilli, A. Sabharwal, and B. Aazhang, "Design of multiple antenna coding schemes with channel feedback," in Proc. 35th Asilomar Conference on Signals, Systems and Computers, pp. 1009-1013, Nov. 2001. 


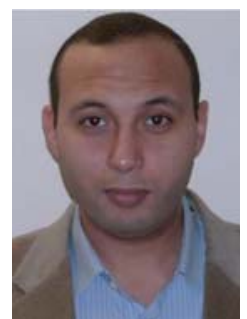

Ahmed K. Sadek (M'07) received the B.Sc. degree (with highest Honors) and the M.Sc. degree in electrical engineering from Alexandria University, Alexandria, Egypt in 2000 and 2003, respectively. He received the Ph.D. degree in electrical engineering from the University of Maryland, College Park, in 2007. He is currently a Senior Engineer with Corporate Research and Development, Qualcomm Incorporated. His current research interests are in the areas of cognitive radios, spectrum sharing, interference management, cooperative communications, and

MIMO-OFDM systems.

In 2000, Dr. Sadek won the first prize in IEEE Egypt Section undergraduate student contest for his B.Sc. graduation project. He received the Graduate School Fellowship from the University of Maryland in 2003 and 2004, and the Distinguished Dissertation Fellowship award from the Department of Electrical Engineering, University of Maryland in 2007. He is a co-author of Cooperative Communications and Networking (Cambridge University Press, 2008).

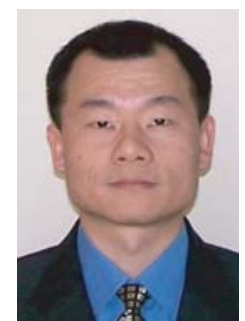

Weifeng Su (M'03) received the Ph.D. degree in electrical engineering from the University of Delaware, Newark in 2002. He received his B.S. and Ph.D. degrees in applied mathematics from Nankai University, Tianjin, China, in 1994 and 1999 respectively. His research interests span a broad range of areas from signal processing to wireless communications and networking, including spacetime coding and modulation for MIMO wireless communications, MIMO-OFDM systems, cooperative communications for wireless networks, and ultra-wideband (UWB) communications.

Dr. Su has been an Assistant Professor at the Department of Electrical Engineering, State University of New York (SUNY) at Buffalo since March 2005. From June 2002 to March 2005, he was a Postdoctoral Research Associate with the Department of Electrical and Computer Engineering and the Institute for Systems Research (ISR), University of Maryland, College Park. Dr. Su received the Signal Processing and Communications Faculty Award from the University of Delaware in 2002 as an outstanding graduate student in the field of signal processing and communications. In 2005, he received the Invention of the Year Award from the University of Maryland. Dr. $\mathrm{Su}$ has been an Associate Editor of IEEE TRANSACTIONS ON VEHICULAR Technology, IEEE Signal Processing LetTers, and a Guest Editor of special issue on cooperative communications and networking of IEEE Journal of SELECTED AREAS IN COMMUNICATIONS.

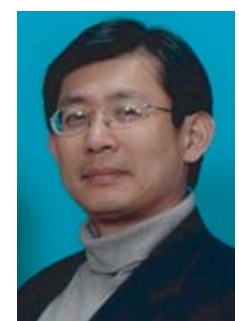

K. J. Ray Liu (F'03) is Distinguished ScholarTeacher of University of Maryland, College Park. He leads the Maryland Signals and Information Group conducting research encompassing broad aspects of information technology including communications and networking, information forensics and security, multimedia signal processing, biomedical imaging, and bioinformatics.

Dr. Liu is the recipient of numerous honors and awards including best paper awards from IEEE Signal Processing Society (twice), IEEE Vehicular Technology Society, and EURASIP; IEEE Signal Processing Society Distinguished Lecturer, EURASIP Meritorious Service Award, and National Science Foundation Young Investigator Award. He also received various teaching and research recognitions from University of Maryland including university-level Invention of the Year Award and both Outstanding Research Award and Poole and Kent Senior Faculty Teaching Award from A. James Clark School of Engineering Faculty.

Dr. Liu is Vice President-Publications and on the Board of Governor of IEEE Signal Processing Society. He was the Editor-in-Chief of IEEE SIGNAL PROCESSING MAGAZINE and the founding Editor-in-Chief of EURASIP JOURNAL ON APPLIED SIGNAL PROCESSING.

His recent books include Cooperative Communications and Networking (Cambridge University Press, 2008); Resource Allocation for Wireless Networks: Basics, Techniques, and Applications (Cambridge University Press, 2008); Ultra-Wideband Communication Systems: The Multiband OFDM Approach (IEEE-Wiley, 2007); Network-Aware Security for Group Communications (Springer, 2007); Multimedia Fingerprinting Forensics for Traitor Tracing (Hindawi, 2005). 